\title{
Modelo de auditoría para evaluar la gestión de mantenimiento de activos físicos
}

\section{Audit Model To Assess Physical Assets Maintenance Management}

\author{
Mariela Fernanda Chang Parrales. ${ }^{1}$, Sergio Raúl Villacrés Parra. ${ }^{2}$, Mayra Alexandra Viscaíno
} Cuzco. ${ }^{3}$ \& César Marcelo Gallegos Londoño. ${ }^{4}$

\section{Recibido: 05-01-2020 / Revisado: 02-02-2020 /Aceptado: 18-02-2020/ Publicado: 07-03-2020}

\begin{abstract}
.
DOI: https://doi.org/10.33262/concienciadigital.v3i1.2.1189

The maintenance audit allows to identify opportunities for improvement in the activities carried out by a company to maintain or restore physical assets in the operating conditions desired by its users. The proposal of the present work is to provide a tool that allows to apply a maintenance audit to any type of organization such as industrial companies, hospitals, and others. The proposed audit process consists of four phases: planning, doing, verifying and acting, in accordance with the guidelines of ISO 19011. The criteria of the evaluation instrument were defined by the Delphi technique and subsequently weighted using the multicriteria analysis technique called Analytic Hierarchy Process (AHP). Once the questionnaire was determined, it was executed in accordance with the planning and a diagnosis was made of the state of maintenance management of a cement company in Ecuador, obtaining 62 conformities and three nonconformities as a result of the audit, so the company an evaluation of $92 \%$, equivalent to a "cuasi satisfactory" maintenance management. In addition, it will be identified that there is potential to improve aspects related to: maintenance human resources, maintenance management control, maintenance planning and programming, to obtain $100 \%$ conformities.

1 Ingeniera en Mantenimiento en libre ejercicio, marielafernanda_15@hotmail.es

2 Escuela Superior Politécnica de Chimborazo, Facultad de Mecánica, Grupo de investigación "Organización del Mantenimiento", Riobamba, Ecuador, sergio.villacres@espoch.edu.ec

${ }^{3}$ Escuela Superior Politécnica de Chimborazo, Facultad de Mecánica, Grupo de investigación "Organización del Mantenimiento", Riobamba, Ecuador, mayra.viscaino@espoch.edu.ec

${ }^{4}$ Escuela Superior Politécnica de Chimborazo, Facultad de Mecánica, Grupo de investigación "Organización del Mantenimiento", Riobamba, Ecuador, cesar.gallegos@espoch.edu.ec
\end{abstract}


Keywords: Audit, Management, Maintenance, Criteria, Audit.

\section{Resumen.}

La auditoría de mantenimiento permite identificar las oportunidades de mejora en las actividades que esté realizando una empresa para mantener o restaurar a los activos físicos a las condiciones de funcionamiento deseadas por sus usuarios. La finalidad del presente trabajo es proporcionar una herramienta que permita aplicar una auditoría de mantenimiento a cualquier tipo de organización como por ejemplo empresas industriales, hospitales entre otros. El proceso de auditoría propuesto consta de cuatro fases: planear, hacer, verificar y actuar, de acuerdo con las directrices de la norma ISO 19011. Los criterios del instrumento de evaluación se definieron mediante la técnica Delphi y posteriormente se ponderaron mediante la técnica de análisis multicriterio denominada Analytic Hierarchy Process (AHP). Determinado el cuestionario a utilizarse se lo ejecutó de acuerdo con la planificación y se realizó un diagnóstico del estado de la gestión de mantenimiento de una empresa cementera del Ecuador, obteniéndose como resultados de la auditoría 62 conformidades y tres no conformidades, por lo que la empresa alcanzó una valoración del 92\%, equivalente a una gestión de mantenimiento "cuasi satisfactoria". Además, se logró identificar que existe potencial para mejorar aspectos relacionados con: recursos humanos de mantenimiento, control de la gestión de mantenimiento, planificación y programación de mantenimiento, para obtener el 100\% de conformidades.

Palabras claves: Auditoría, Gestión, Mantenimiento, Criterios, Auditoría.

\section{Introducción.}

El mantenimiento está catalogado como una herramienta que, combinada con actividades técnicas, administrativas y de gestión contribuye a que los activos cumplan la función requerida establecida por el usuario (EN13306:2018, 2018), (Mwanza \& Mbohwa, 2015). Últimamente la gestión de mantenimiento se ha convertido en un eje fundamental para la industria de cualquier tipo, debido a que entre sus objetivos están, asegurar la disponibilidad de un equipo para cumpla la función requerida a un costo óptimo considerando la seguridad de las personas y el impacto sobre el medio ambiente, además de mantener la durabilidad del equipo y la calidad del producto o servicio (EN13306:2018, 2018), (Orozco, Narváez, García, \& Quintero, 2017). La gestión de mantenimiento está constituida de áreas funcionales que la articulan, independientemente del activo sujeto de mantenimiento; entre éstas se encuentran: organización general del mantenimiento; recursos humanos; control económico; planificación, programación y control; ingeniería de mantenimiento; tercerización; gestión de seguridad (Acosta \& Troncoso, 2011, citado en Chang, 2019).

Con el objetivo de determinar si las actividades que se han realizado en las áreas funcionales, se 
han ejecutado conforme estándares deseados, y garantizar que los productos o servicios sean de calidad, es necesario realizar una auditoría de mantenimiento que evidencie el grado de cumplimiento de las exigencias.

Una auditoría puede ser interna o externa y éstas últimas se realizan a proveedores o pueden ser auditorías de tercera parte, las cuales se llevan a cabo con fines de certificación, según lo indica la norma ISO 19011; además ésta misma norma define a una auditoría como un "proceso sistemático, independiente y documentado para obtener evidencias de la auditoría y evaluarlas de manera objetiva con el fin de determinar la extensión en que se cumplen los criterios de auditoría. Los responsables de llevar acabo la auditoría, es el denominado grupo auditor, que debe estar conformado por personal profesionalmente idóneo, de alta competitividad (Sánchez \& Calderón, 2013), con conocimiento y experiencia en el área técnica, en este caso en gestión del mantenimiento; según (Rodríguez Rieiro et al., 2010), existen tres aspectos que para los auditores supone mayor dificultad como: la revisión previa de la documentación, la elaboración del informe y la auditoría de los procesos operativos. Un aspecto relevante, que se debe garantizar es que el grupo auditor debe mantener un comportamiento ético, imparcial y objetivo durante todo el tiempo que dure el proceso (CGE, 2003), que garantice que los resultados reflejen la verdadera condición del área o entidad auditada y sean conducentes a la mejora.

Cuando se habla de "proceso" se debe entender que una auditoría, consta de una serie de etapas, la norma ISO 19011:2011 aplica la filosofía del círculo PHVA que corresponde a: planificar, hacer, verificar y actuar (ISO 19011, citado en Chang, 2019); para la contraloría general del estado del Ecuador, organismo que realiza auditorías gubernamentales, aplica las etapas de: planificación (preliminar y específica), ejecución del trabajo y comunicación de resultados (CGE, 2003), generalmente la ejecución del trabajo consiste en la revisión documental y búsqueda de evidencias que corroboren o no el cumplimiento de un hecho, la norma ISO 19011 cataloga a esto como "evidencia de la auditoría" que son los registros, declaraciones de hechos o cualquier otra información que sean pertinentes para los criterios de auditoría y que sean verificables, cuando la evidencia de auditoría cumple con lo exigido por el criterio o requisito se produce una "conformidad", caso contrario una "no conformidad" (ISO 19011, 2011, citado en Chang, 2019). Por otra parte, la comunicación de resultados se lo lleva a cabo mediante la emisión de un informe (Rodríguez Rieiro et al., 2010). Fruto de la ejecución del proceso de auditoría, se generarán datos importantes sobre la situación o el objeto auditado, denominados "hallazgos"(Restrepo-Medina, 2018) que se refieren a hechos, asuntos, debilidades o deficiencias detectadas que deben ponerse en conocimiento de las autoridades o funcionarios de la entidad auditada (CGE, 2003), para que se realicen los análisis y evaluaciones correspondientes y se tomen las medidas correctivas.

Generalmente, en cada auditoría hay cierta subjetividad y esto es cierto especialmente en la auditoría de mantenimiento, ya que no existe un estándar como ISO 9001: 2000, para el caso de los sistemas de gestión de calidad. Sin embargo, las auditorías de mantenimiento pueden llevarse 
a cabo y sus resultados constituyen un aporte válido para la gestión auditada (Grencki \& Legát, 2007).

Conocer las áreas funcionales en el área de mantenimiento y sus componentes, permiten definir los criterios de la auditoría; un criterio de auditoría, según la norma ISO 19011, es el "Grupo de políticas, procedimientos o requisitos usados como referencia y contra los cuales se compara la evidencia de auditoría". Definir los criterios que servirán como la medida estándar de cumplimiento, para el área de mantenimiento; es un punto crucial dentro de una auditoría y deben ser seleccionados previo a la planificación de la auditoría, en este sentido las normas aportan valiosos estándares; en lo referente a mantenimiento se han encontrado normas como: Manual para evaluar los sistemas de mantenimiento en la industria (COVENIN 2500, 1993) y "Sistemas de gestión de mantenimiento - requisitos" (NB 12017, 2013), además trabajos desarrollados en el ámbito de auditorías de mantenimiento.

(Acosta y Troncoso, 2011), en su investigación sobre auditorías a hospitales, han establecido áreas funcionales que contienen criterios de evaluación para auditorías de mantenimiento, como: organización de mantenimiento, recursos humanos, control económico, planificación, programación y control, ingeniería de mantenimiento, tercerización y gestión de seguridad. Mientras que algunas normas internacionales indican que se deben evaluar aspectos relacionado con: actividades de mantenimiento, mantenimiento preventivo, mantenimiento por avería, apoyo logístico y recursos (COVENIN 2500, 1993), requisitos generales, de la alta gerencia, de la planeación administrativa y operativa, requisitos del cliente, de prestación de servicios y de la ejecución (NB 12017, 2013).

Las valoraciones que se realizan dentro de la verificación del cumplimiento de los criterios puede ser una valoración cualitativa o cuantitativa, para este último caso se requiere conocer cuál es el criterio más importante dentro de todos los criterios que serán evaluados en la auditoría; para solventar este requerimiento se puede recurrir a los diferentes métodos de análisis multicriterio (Carnero \& Gómez, 2016), en este caso una metodología ampliamente usada con éstos fines, es la metodología Analytic Hierarchy Process, conocida como AHP por sus siglas en inglés. Esta metodología resume cuantitativamente la opinión de expertos, a quienes se debe recurrir para que presenten su valoración y ha sido usado por otras investigaciones con estos mismos propósitos (Viscaíno Cuzco, Villacrés Parra, Gallegos Londoño, \& Negrete Costales, 2019), (Pecchia \& Melillo, 2013), (Viscaíno, Quesada, \& Villacrés, 2017).

El objetivo de este trabajo es establecer una metodología de auditoría que conjuga una evaluación cuantitativa y cualitativa para valorar la gestión de mantenimiento en el área industrial, la metodología será aplicada a un caso de estudio de modo que se puede verificar su aplicabilidad.

\section{Metodología}

La metodología empleada para desarrollar esta investigación corresponde al tipo cualitativo y está 
estructurada en tres fases, en la primera se realizó la selección y ponderación de los criterios a auditar, en la segunda fase el desarrollo de la metodología de auditoría y finalmente la validación de la metodología, con aplicación a un caso de estudio, en los siguientes párrafos se expone con mayor detalle cada fase.

FASE 1: Los criterios a evaluar en la auditoría de mantenimiento se seleccionaron mediante la aplicación de la técnica Delphi, mediante una encuesta dirigida a los estudiantes de maestría de la Escuela Superior Politécnica de Chimborazo (ESPOCH) y con experiencia en gestión de mantenimiento, en la cual manifestaron su aprobación o rechazo a los criterios y subcriterios enunciados, así como la posibilidad de proponer nuevos aspectos a evaluar.

La Tabla $\mathrm{N}^{\circ} 1$ es un extracto de la encuesta, la misma que exponía los siete criterios de auditoría y los subcriterios, cada uno con un casillero para que el encuestado pueda marcar su opinión.

Tabla $\mathbf{N}^{\circ}$ 1: Criterio de auditoría organización de mantenimiento

\begin{tabular}{|l|l|}
\hline Subcriterios & Observaciones \\
\hline a. Políticas de mantenimiento. & a. \\
b. Herramienta informática para la gestión & b. \\
$\quad$ de mantenimiento. & c. \\
c. Organigrama. & \\
\hline Propuestas & \\
\hline & \\
\hline
\end{tabular}

Elaborado por: autores

Luego se realizó un análisis de los resultados obtenidos, con docentes de la carrera de Mantenimiento Industrial de la ESPOCH y finalmente se definieron los criterios y subcriterios resultando un total de siete criterios y 22 subcriterios.

La ponderación se realizó usando la metodología AHP que contiene cuatro pasos (Aznar \& Guijarro, 2012):

1. Definir el objetivo o problema a resolver.

2. Definir la estructura jerarquizada de decisión.

3. Construir las matrices de juicios por partes.

4. Determinar las prioridades de los criterios.

Primer paso: se definió el objetivo de aplicar la metodología AHP, que fue priorizar los criterios 
de auditoría de mantenimiento: organización del mantenimiento, recursos humanos, control de la gestión, planificación y programación, mantenimiento correctivo, contratación externa de mantenimiento y manejo de inventario de bodega para mantenimiento (Acosta \& Troncoso, 2011, citado en Chang, 2019) el segundo nivel corresponde a los criterios de la auditoría y en el tercer nivel se encuentran los subcriterios,.

Segundo paso: se estructuró la jerarquía de los elementos a ponderar, la figura $\mathrm{N}^{\circ} 1$ muestra un organizador gráfico compuesto de un primer nivel que indica el objetivo de la aplicación de la metodología AHP, el segundo y tercer nivel corresponden a criterios y subcriterios respectivamente, se han codificado para identificarlos.

Figura $N^{\circ}$ 1. Estructura jerárquica

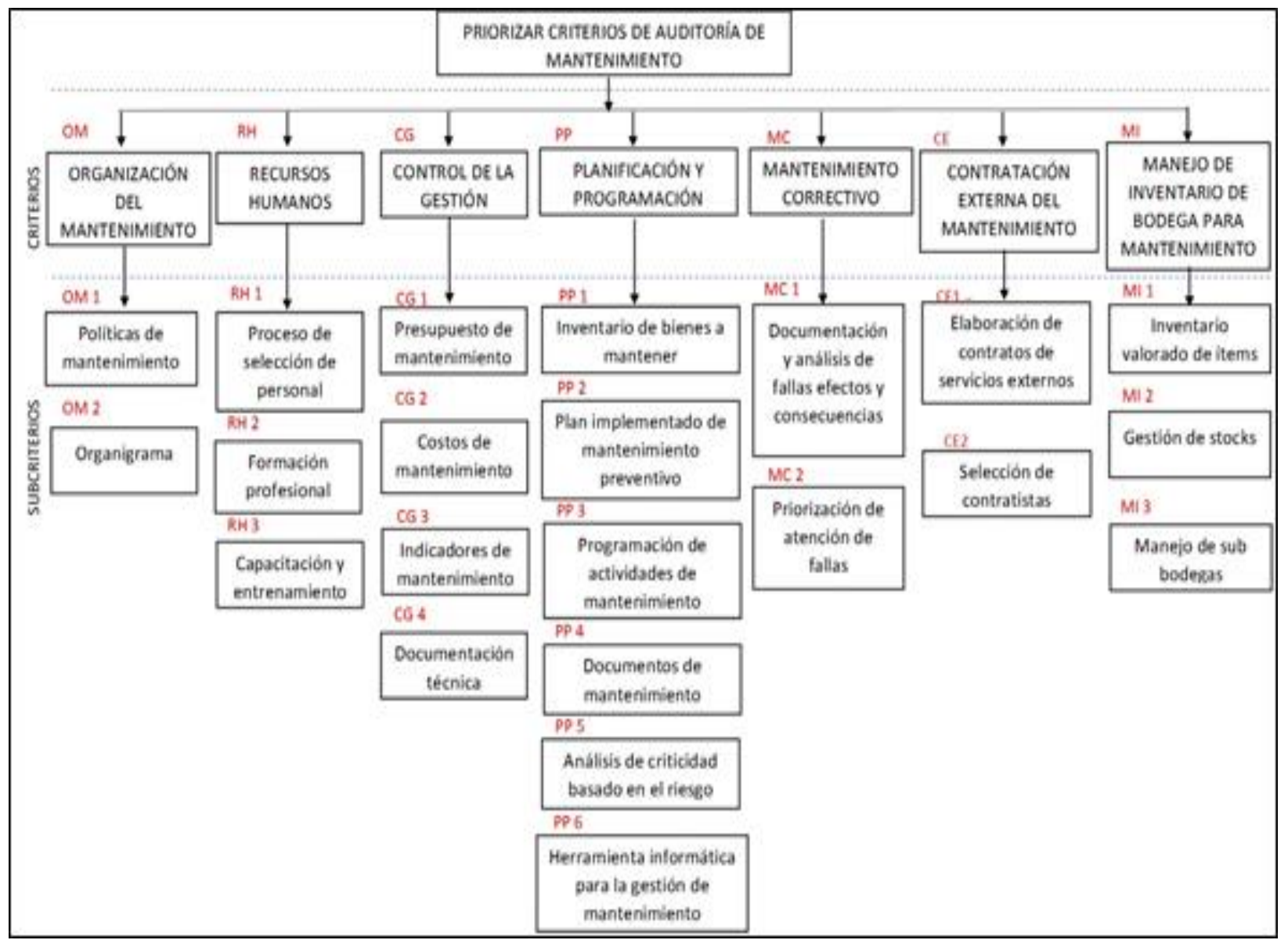

Fuente: Autores

Elaborado por: autores

Tercer paso: Se buscó el criterio de 30 especialistas en el área de mantenimiento, de los cuales cinco poseen grado de magíster en gestión de mantenimiento, 23 estudiantes de maestría de gestión de mantenimiento y dos ingenieros de mantenimiento a quienes se les solicitó valorar la 
importancia de los criterios que intervendrán en la auditoría de mantenimiento, con los resultados de las valoraciones se elaboraron las matrices de comparación pareada que permitieron calcular el grado de importancia de los criterios y subcriterios ponderados. En la tabla $\mathrm{N}^{\circ} 2$ se indica el instrumento aplicado, el cual contiene los niveles de importancia acompañados de una escala numérica del 1 al 9, cada encuestado valoró el grado de importancia de un criterio respecto a otro según su conocimiento y experiencia en el área de gestión de mantenimiento.

Tabla $N^{\circ}$ 2: Matriz de comparación

\begin{tabular}{|c|c|c|c|c|c|c|c|c|c|c|c|c|c|c|c|c|c|c|}
\hline \multicolumn{19}{|c|}{\begin{tabular}{|l} 
MATRIZ DE COMPARACIÓN PAREADA DE CRITERIOSDE MANTENIMIENTO \\
\end{tabular}} \\
\hline \multirow{3}{*}{ CRITERIOS } & \multicolumn{8}{|c|}{ Importancia } & \multirow[b]{2}{*}{ 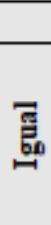 } & & \multirow[b]{2}{*}{ 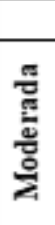 } & \multirow{2}{*}{\multicolumn{2}{|c|}{ 㹂 }} & \multirow{2}{*}{\multicolumn{2}{|c|}{ 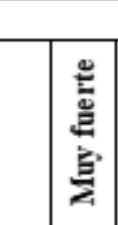 }} & \multirow{2}{*}{\multicolumn{2}{|c|}{ 氙 }} & \multirow{3}{*}{ 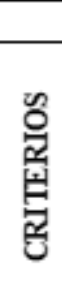 } \\
\hline & 吾 & & 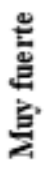 & & 这 & & 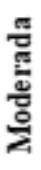 & & & & & & & & & & & \\
\hline & 9 & 8 & 7 & 6 & 5 & 4 & 3 & 2 & 1 & 2 & 3 & 4 & 5 & 6 & 7 & 8 & 9 & \\
\hline \multirow{6}{*}{$\begin{array}{l}\text { OM Organización } \\
\text { general del } \\
\text { mantenimiento }\end{array}$} & & & & & & & & & & & & & & & & & & $\mathrm{RH}$ \\
\hline & & & & & & & & & & & & & & & & & & CG \\
\hline & & & & & & & & & & & & & & & & & & PP \\
\hline & & & & & & & & & & & & & & & & & & $\mathrm{MC}$ \\
\hline & & & & & & & & & & & & & & & & & & $C E$ \\
\hline & & & & & & & & & & & & & & & & & & $\mathrm{M}$ \\
\hline \multirow{5}{*}{$\begin{array}{l}\mathrm{RH}: \text { Recursos } \\
\text { humanos de } \\
\text { mantenimiento }\end{array}$} & & & & & & & & & & & & & & & & & & $C G$ \\
\hline & & & & & & & & & & & & & & & & & & PP \\
\hline & & & & & & & & & & & & & & & & & & $\mathrm{MC}$ \\
\hline & & & & & & & & & & & & & & & & & & $\mathrm{CE}$ \\
\hline & & & & & & & & & & & & & & & & & & $\mathrm{M}$ \\
\hline \multirow{4}{*}{$\begin{array}{l}\text { CG. Control de la } \\
\text { gestión }\end{array}$} & & & & & & & & & & & & & & & & & & $\mathrm{PP}$ \\
\hline & & & & & & & & & & & & & & & & & & $\mathrm{MC}$ \\
\hline & & & & & & & & & & & & & & & & & & $\mathrm{CE}$ \\
\hline & & & & & & & & & & & & & & & & & & $\mathrm{M}$ \\
\hline \multirow{3}{*}{$\begin{array}{l}\text { PP: Planificación y } \\
\text { programación }\end{array}$} & & & & & & & & & & & & & & & & & & $\mathrm{MC}$ \\
\hline & & & & & & & & & & & & & & & & & & $\mathrm{CE}$ \\
\hline & & & & & & & & & & & & & & & & & & $\mathrm{M}$ \\
\hline \multirow{2}{*}{$\begin{array}{l}\mathrm{MC}: \text { Mantenimiento } \\
\text { Correctivo }\end{array}$} & & & & & & & & & & & & & & & & & & $\mathrm{TM}$ \\
\hline & & & & & & & & & & & & & & & & & & $\mathrm{M}$ \\
\hline $\begin{array}{l}\text { CE: Contratación } \\
\text { externa de } \\
\text { mantenimiento }\end{array}$ & & & & & & & & & & & & & & & & & & $\mathrm{M}$ \\
\hline
\end{tabular}

Elaborado por: autores

Cuarto paso: el último paso de la metodología AHP consistió en el procesamiento de los datos obtenidos mediante la matriz de comparación pareada que genera los pesos ponderados de los criterios y subcriterios de auditoría de la gestión de mantenimiento.

FASE 2: El proceso de auditoría consta de: planificar, hacer, verificar y actuar (ISO 19011, 2011) 
Planificar: consistió en la planificación de los elementos necesarios para realizar la auditoría (Galar, Kumar, Aditya, \& Berges, 2011). Los aspectos que se establecieron en el programa de auditoría, se explican en la Tabla $\underline{N^{\circ}} 3$.

Tabla $N^{\circ}$ 3: Programa de auditoría

ELEMENTOS DEL PROGRAMA
DETALLE

Los objetivos del programa de auditoría tienen la finalidad de guiar la ejecución de las Objetivo del programa de auditorías contempladas dentro del programa de auditorías. La elaboración del objetivo u auditoría objetivos del programa deben tomar en cuenta aspectos como el nivel de implementación del sistema de gestión a auditarse y los resultados de auditorías previas.

Responsabilidades del encargado del programa de auditoría

Competencia del encargado del programa de auditoría auditoría

Procedimientos para el programa de auditoría

Recursos del programa de auditoría:
La responsabilidad principal del encargado de auditorías es la elaboración del programa para una o varias auditorías en su totalidad de manera que se asegure el cumplimiento de todo lo estipulado en el programa.

Las habilidades que son necesarias para gestionar el programa de auditoría son:

conocimiento de los elementos que intervienen en la programación de la auditoría, competencia profesional para el manejo de la información.

El alcance del programa de auditoría se encuentra establecido por el tamaño de la organización auditada y el desarrollo de los sistemas de gestión que serán revisados. Es posible que el programa de auditorías para un periodo determinado de tiempo incluya solamente una auditoría, aquel caso es correcto y también se deben cumplir con todos los pasos de la metodología de la auditoría.

Para lograr una correcta ejecución del programa auditoría es necesario garantizar el cumplimiento de los principios éticos de confidencialidad e integridad por parte del equipo auditor por lo que cabe establecer acuerdos de manejo de información por ambas partes involucradas en la auditoría. Otro procedimiento que se debe definir es el método de muestreo para realizar la recolección de las evidencias durante la auditoría el mismo que puede ser: muestreo basado en el juicio, muestreo estadístico.

La gestión previa de los recursos del programa la debe realizar la persona encargada del mismo, de manera que sea posible alistar todos los elementos necesarios para realizar las auditorías programadas en una organización. Los recursos que interviene en el programa de auditoría son:

Recurso humano: equipo auditor, guía, personal involucrado en la organización auditada.

Documentación: programa de auditoría, plan de auditoría, evidencias de auditoría.

Recursos financieros: costos del equipo auditor, hospedajes, viáticos, entre otros.

Fuente: (ISO 19011, 2011, citando en Chang, 2019)

Elaborado por: autores

Hacer: corresponde a la aplicación del plan de auditoría que debe contener los siguientes elementos: objetivo de la auditoría, alcance de auditoría, composición del equipo auditor, actividades y recursos. 
ISSN: 2600-5859

Vol. 3, N¹.2, p. 104-122, marzo, 2020

Parte del proceso de auditoría es la revisión documental de evidencias que consiste en contrastar lo solicitado en el instrumento de evaluación con las evidencias presentadas para realizar el levantamiento de los hallazgos.

Para la generación de hallazgos se debe seguir proceso de revisión documental (ver Figura $\mathrm{N}^{\circ} 2$ ).

Figura $N^{\circ}$ 2. Proceso de revisión documental

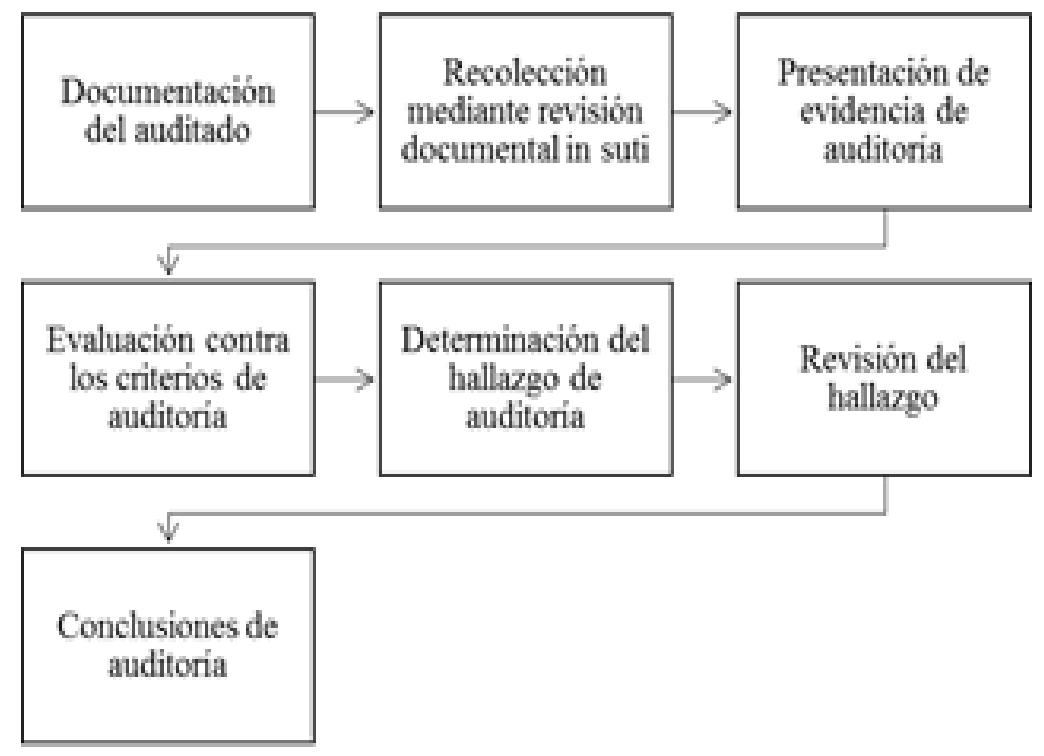

Fuente: (Chang, 2019)

Al cumplimiento de los criterios exigidos, le corresponde una valoración cuantitativa y cualitativa, que indica en qué nivel se encuentre la gestión de mantenimiento auditada según la escala de la Figura $\underline{\mathrm{N}^{\circ}} 3$.

Figura $\mathbf{N}^{\circ}$ 3. Niveles de cumplimiento

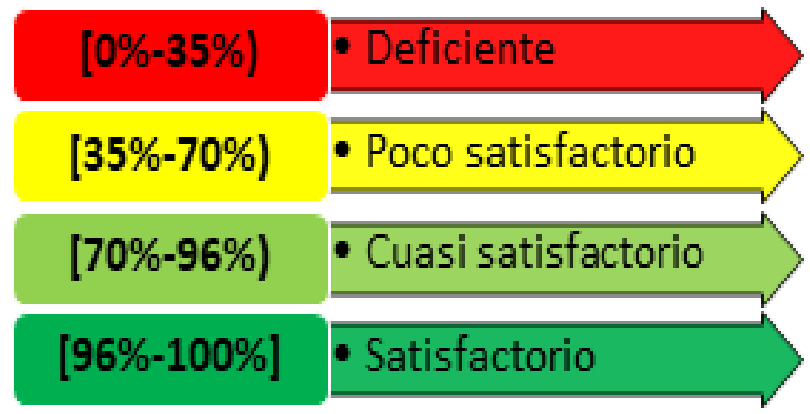

Fuente: Elaboración propia 
Verificar: Esta etapa corresponde al monitoreo del programa de auditoría, en especial el cumplimiento del cronograma elaborado para la realización de la auditoría. En este punto es posible realizar variaciones en el programa de auditoría conforme a los hallazgos de auditoría. (Acosta \& Troncoso, 2011, citando en Chang, 2019)

Actuar: El último paso que contiene el programa de auditoría fue la retroalimentación en base a los resultados obtenidos durante la aplicación del programa. En esta etapa valoró los logros obtenidos y si cumplió con el objetivo planteado del programa de manera que fue posible detectar falencias en las actividades realizadas e identificación de aspectos que permitan mejorar el programa de auditoría (Chang, 2019).

FASE 3: corresponde a validación de la metodología, con aplicación a un caso de estudio para tal propósito se realizó un muestreo intencional, seleccionando a una empresa cementera del Ecuador con aproximadamente 60 años de funcionamiento cuya actividad económica es la producción de cemento, a partir de caliza y otras materias primas para su posterior comercialización en sacos y a granel. Por la complejidad del proceso de producción, la gestión del mantenimiento es un apoyo fundamental para el desarrollo de las actividades productivas de la empresa (Chang, 2019).

\section{Resultados}

Posterior a la aplicación de la primera fase de la metodología propuesta, se seleccionaron los criterios y subcriterios, usando la técnica Delphi, los mismos que se enuncian en la tabla $\underline{N}^{\circ} 4$. La aplicación de la metodología AHP dio como resultado los porcentajes de calificación de los criterios de auditoría como se muestra en la tabla $\mathrm{N}^{\circ} 4$, en donde se valoran los siguientes criterios:

Organización del mantenimiento (OM): El compromiso con la gestión del mantenimiento inicia con la alta gerencia de una empresa y la parte administrativa del mantenimiento es la encargada de establecer las políticas bajo las cuales se regirán las actividades del departamento logrando que el personal realice sus actividades de forma sinérgica. Un elemento importante que ayuda a visualizar la estructura funcional del área de mantenimiento es el organigrama. La participación continua de la gerencia es primaria para proporcionar orientación y dirección a la función de mantenimiento (Cholasuke, Bhardwa, \& Antony, 2004, citado en Chang, 2019).

Recursos humanos (RH): Una correcta estructura jerárquica de una organización, la existencia de herramientas tecnológicas y una adecuada logística no son garantías del buen desempeño del talento humano que conforma una institución. Es por lo que la organización del mantenimiento se encuentra respaldada en un equipo de trabajo capacitado y motivado. (Bana, Carmen, \& Duarte, 2012, citado en Chang, 2019). Para lograrlo son necesarios procesos rigurosos de reclutamiento y selección de personal en la cantidad idónea tomando en cuenta las cargas de trabajo que se asignarán a cada individuo. Para mejorar continuamente la realización de las tareas de mantenimiento es importante un sistema de formación permanente del personal de acuerdo con las 
necesidades de capacitación que se identifiquen (Chang, 2019).

Control de la gestión (CG): Para conocer la factibilidad de las políticas empresariales planteadas y con el ánimo de mejorar la gestión que se realiza alrededor del mantenimiento es indispensable el cálculo de indicadores que revelen los logros obtenidos y los aspectos que requieren mejoras. Las actividades de control y seguimiento aseguran que el sistema de gestión de mantenimiento se encuentre en constante revisión y cada vez que se alcancen las metas planteadas se formulen nuevos objetivos para el futuro, obteniéndose así, la mejora continua de procesos. (Espinosa \& Salinas, 2010, citado en Chang, 2019).

Planificación y programación (PP): Una de las estrategias para obtener éxito en la gestión de activos físicos es la planificación y programación de cada actividad que se haya definido bajo las estrategias de mantenimiento que se apliquen a cada uno de los activos físicos de una institución. La aplicación de actividades de mantenimiento planificadas tiene la finalidad de reducir la probabilidad de falla en un activo. La función del programa de mantenimiento de la planta es preservar y restaurar la seguridad inherente, la fiabilidad y la disponibilidad de la planta incluyendo estructuras, sistemas y componentes para una operación confiable y segura (Vaisnys, Contri, Rieg, \& Bieth, 2016, citado en Chang, 2019)

Mantenimiento Correctivo (MC): Este mantenimiento se aplica posterior a la ocurrencia de una falla en un activo, el mantenimiento correctivo no es únicamente subsanar fallas que ocurran en un determinado activo, sino que es conveniente realizar un análisis por el cual ocurren las fallas imprevistas y así evitar que se repitan. Una correcta gestión del mantenimiento incluye que se programe toda la logística necesaria para realizar una actividad correctiva de manera que el tiempo de intervención por mantenimiento sea óptimo y la reparación sea de calidad (Mora \& Arango, 2010, citado en Chang, 2019).

Contratación externa de mantenimiento (CE): Se denomina contratación externa al hecho de que una empresa contrate los servicios de mantenimiento bajo condiciones prestablecidas. Un punto clave a tomar en cuenta cuando una organización opta por la contratación externa del mantenimiento es la supervisión de las actividades estipuladas en el contrato. El objetivo de evaluar la tercerización de mantenimiento es conocer si se obtienen beneficios de esta actividad o si al contrario es una actividad ineficaz (Jiménez \& Valencia, 2012, citado en Chang, 2019).

Manejo de inventario de bodega para mantenimiento (MI): La logística de los repuestos para mantenimiento es un apoyo fundamental que permite el desarrollo continuo de las actividades que fueron planeadas y programadas en los activos de una instalación (Viveros, Stegmaier, Kristjanpoller, Barbera, \& Crespo, 2013, citado en Chang, 2019). Debido a que los valores económicos que se poseen en una bodega de mantenimiento son elevados, es muy importante llevar un control de existencias y de los niveles de requerimientos del departamento de mantenimiento debido a que una correcta gestión de inventario de repuestos permite a las empresas 
ahorrar recursos económicos (Chang, 2019).

Tabla N 4: Pesos de los criterios de auditoría

\begin{tabular}{|c|c|c|c|c|c|}
\hline \multicolumn{2}{|r|}{ CRITERIOS } & PESO & \multicolumn{2}{|r|}{ SUBCRITERIOS DE EVALUACION } & \multirow{2}{*}{$\begin{array}{c}\text { PESO } \\
0,72 \\
\end{array}$} \\
\hline \multirow{2}{*}{$\mathbf{O M}$} & \multirow{2}{*}{$\begin{array}{l}\text { Organización del } \\
\text { mantenimiento }\end{array}$} & \multirow{2}{*}{$21 \%$} & OMI & Politicas de mantenimiento & \\
\hline & & & OM2 & Organigrama & 0,28 \\
\hline \multirow{3}{*}{ RH } & \multirow{3}{*}{$\begin{array}{l}\text { Recursos humanos de } \\
\text { mantenimiento }\end{array}$} & \multirow{3}{*}{$17 \%$} & RH1 & Proceso de selección de personal & 0,23 \\
\hline & & & RH2 & Formación profesional & 0,32 \\
\hline & & & RH3 & Capacitación y entrenamiento & 0,45 \\
\hline & & & & & \\
\hline \multirow{4}{*}{ CG } & \multirow{4}{*}{ Control de la gestión } & \multirow{4}{*}{$20 \%$} & CG1 & Presupuesto de mantenimiento & 0,23 \\
\hline & & & CG2 & Costos de mantenimiento & 0,18 \\
\hline & & & CG3 & Indicadores de mantenimiento & 0,30 \\
\hline & & & CG4 & Documentación técnica & 0,29 \\
\hline & & & & & \\
\hline \multirow{6}{*}{$\mathbf{P P}$} & \multirow{6}{*}{$\begin{array}{l}\text { Planificación y } \\
\text { programación }\end{array}$} & \multirow{6}{*}{$18 \%$} & PP1 & Inventario de bienes a mantener. & 0,13 \\
\hline & & & PP2 & $\begin{array}{l}\text { Plan implementado de mantenimiento } \\
\text { preventivo }\end{array}$ & 0,31 \\
\hline & & & PP3 & $\begin{array}{l}\text { Programación de actividades de } \\
\text { mantenimiento }\end{array}$ & 0,22 \\
\hline & & & PP4 & Documentos de mantenimiento & 0,10 \\
\hline & & & PP5 & $\begin{array}{l}\text { Analisis de criticidad basado en el } \\
\text { riesgo }\end{array}$ & 0,12 \\
\hline & & & PP6 & $\begin{array}{l}\text { Herramienta informática para la gestión } \\
\text { de mantenimiento }\end{array}$ & 0,12 \\
\hline \multirow{3}{*}{ MC } & \multirow{3}{*}{$\begin{array}{l}\text { Mantenimiento } \\
\text { correctivo }\end{array}$} & \multirow{3}{*}{$10 \%$} & & & \\
\hline & & & MC1 & $\begin{array}{l}\text { Documentación y análisis de fallas } \\
\text { efectos y consecuencias }\end{array}$ & 0,67 \\
\hline & & & MC2 & Priorización de atención de fallas & 0,33 \\
\hline \multirow{3}{*}{$\mathbf{C E}$} & \multirow{3}{*}{$\begin{array}{l}\text { Contratación externa } \\
\text { del mantenimiento }\end{array}$} & \multirow{3}{*}{$6 \%$} & & & \\
\hline & & & CE1 & externos & 0,52 \\
\hline & & & CE2 & Selección de contratistas & 0,48 \\
\hline & & & & & \\
\hline \multirow{3}{*}{ MI } & \multirow{3}{*}{$\begin{array}{c}\text { Manejo de inventario } \\
\text { para bodega de } \\
\text { mantenimiento }\end{array}$} & \multirow{3}{*}{$8 \%$} & MII & $\begin{array}{l}\text { Inventario valorado y etiquetado de } \\
\text { items }\end{array}$ & 0,37 \\
\hline & & & MI2 & Gestión de stocks & 0,41 \\
\hline & & & MI3 & Manejo de sub-bodegas & 0,22 \\
\hline
\end{tabular}

Fuente: Elaboración propia

\subsection{Metodología de auditoría}

Mediante la aplicación del circulo PHVA al proceso de auditoría se realizó el plan de auditoría que contempla todas las actividades a ejecutarse antes, durante y después de la auditoría (ver tabla $\mathrm{N}^{\circ}$ 5). Es importante aclarar que la metodología de auditoría que se ejemplifica fue realizada en base a la estructura de la empresa cementera estudiada. Con las actividades definidas se procede a realizar el cronograma de auditoría como se muestra en la tabla $\mathrm{N}^{\circ} 6$, en el cual se define el tiempo estipulado para cada actividad y el personal responsable. Luego de aplicar el instrumento de evaluación se encontraron: 62 "Conformidades", es decir cumplimiento total del requisito exigido y tres "No Conformidades". 
Tabla $N^{\circ}$ 5: Plan de auditoría

\begin{tabular}{|c|c|c|}
\hline \multicolumn{3}{|c|}{ ASPECTOS GENERALES } \\
\hline \multicolumn{3}{|c|}{\begin{tabular}{lll|l|l} 
Objetivos & de & la & Verificar el cumpl \\
auditoría: & & & gestión del manteni
\end{tabular}} \\
\hline \multicolumn{3}{|c|}{\begin{tabular}{ll|l} 
Alcance & de & Unidades de la organización: Mantenimiento \\
auditoría: & Actividades: Gestión de mantenimiento.
\end{tabular}} \\
\hline \multicolumn{3}{|c|}{$\begin{array}{l}\text { Composición del } 1 \text { auditor } \\
\text { equipo auditor }\end{array}$} \\
\hline \multicolumn{3}{|c|}{ DESCRIPCIÓN DE ACTIVIDADES } \\
\hline Nro. & Actividad & Recursos \\
\hline 1 & $\begin{array}{l}\text { Reunión previa: } \\
\text { Revisión de los elementos de auditoría } \\
\text { Aprobación del plan de auditoría. }\end{array}$ & $\begin{array}{l}\text { Equipo auditor } \\
\text { Personal involucrado de la } \\
\text { empresa cementera }\end{array}$ \\
\hline 2 & $\begin{array}{l}\text { Reunión de apertura: } \\
\text { Presentación del equipo auditor. } \\
\text { Confirmación de la aprobación del plan de auditoría por } \\
\text { todos los involucrados. }\end{array}$ & $\begin{array}{l}\text { Instrumento de evaluación } \\
\text { Suministros de oficina } \\
\text { Computadora }\end{array}$ \\
\hline 3 & $\begin{array}{l}\text { Evaluación de criterios: } \\
\text { OM: Organización del mantenimiento } \\
\text { RH: Recursos humanos de mantenimiento } \\
\text { CG: Control de la gestión de mantenimiento } \\
\text { PP: Planificación y programación de mantenimiento } \\
\text { MC: Mantenimiento correctivo } \\
\text { CE: Contratación externa del mantenimiento } \\
\text { MI: Manejo de inventario de bodega para mantenimiento }\end{array}$ & \\
\hline 4 & $\begin{array}{l}\text { Reunión de cierre: } \\
\text { Revisar el cumplimiento de los objetivos y el alcance de la } \\
\text { auditoría. } \\
\text { Exponer los hallazgos de la auditoría. } \\
\text { Presentación de las conclusiones de la auditoría de } \\
\text { mantenimiento. }\end{array}$ & \\
\hline 5 & $\begin{array}{l}\text { Elaboración del informe de auditoría. } \\
\text { Detalle de las no conformidades encontradas. } \\
\text { Distribución del informe de auditoría }\end{array}$ & \\
\hline
\end{tabular}


Fuente: (Chang, 2019)

Tabla $N^{\circ}$ 6: Cronograma de auditoría

\begin{tabular}{|c|c|c|c|}
\hline \multicolumn{4}{|c|}{\begin{tabular}{l|ll} 
Fecha & de $\mathrm{dd} / \mathrm{mm} / \mathrm{aa}$
\end{tabular}} \\
\hline \multicolumn{2}{|c|}{\begin{tabular}{|l|l|}
$\begin{array}{l}\text { Auditoría de gestión de elaboración: } \\
\text { mantenimiento de activos }\end{array}$ & Elaborado por: \\
físicos
\end{tabular}} & Audit & tor líder \\
\hline \multicolumn{4}{|c|}{ Cronograma de actividades } \\
\hline Hora & \multicolumn{2}{|l|}{ Actividad } & Personal Involucrado \\
\hline $8: 30$ & \multicolumn{2}{|l|}{ Reunión de apertura: } & $\begin{array}{l}\text { Auditor } \\
\text { Delegado del área de mantenimiento }\end{array}$ \\
\hline 9:00 & \multicolumn{2}{|l|}{$\begin{array}{l}\text { Evaluación de criterios: } \\
\text { OM Organización del mantenimiento }\end{array}$} & $\begin{array}{l}\text { Auditor } \\
\text { Superintendente de mantenimiento }\end{array}$ \\
\hline $9: 20$ & \multicolumn{2}{|l|}{ RH Recursos humanos de mantenimiento } & $\begin{array}{l}\text { Auditor } \\
\text { Superintendente de mantenimiento } \\
\text { Asistente de talento humano }\end{array}$ \\
\hline $9: 40$ & \multicolumn{2}{|c|}{ CG Control de la gestión de mantenimiento } & $\begin{array}{l}\text { Auditor } \\
\text { Superintendente de mantenimiento } \\
\text { Jefe de mantenimiento programado }\end{array}$ \\
\hline 10:00 & \multicolumn{2}{|c|}{$\begin{array}{l}\text { PP Planificación y programación de } \\
\text { mantenimiento }\end{array}$} & $\begin{array}{l}\text { Auditor } \\
\text { Superintendente de mantenimiento } \\
\text { Jefe de mantenimiento programado } \\
\text { Planeador de paros mayores }\end{array}$ \\
\hline 11:00 & \multicolumn{2}{|l|}{ MC Mantenimiento correctivo } & $\begin{array}{l}\text { Auditor } \\
\text { Superintendente de mantenimiento } \\
\text { Inspector de predictivo mecánico }\end{array}$ \\
\hline $11: 30$ & \multicolumn{3}{|c|}{\begin{tabular}{|lll|l|} 
CE & Contratación & externa & del \\
mantenimiento & Auditor \\
Encargado del departamento de compras
\end{tabular}} \\
\hline $12: 00$ & \multicolumn{3}{|c|}{ MI Manejo de inventario de bodega para|Equipo Auditor } \\
\hline $12: 30$ & \multicolumn{2}{|c|}{$\begin{array}{l}\text { Revisar el cumplimiento de los objetivos y } \\
\text { el alcance de la auditoría. } \\
\text { Exponer los hallazgos de la auditoría. } \\
\text { Presentación de las conclusiones de la } \\
\text { auditoría de mantenimiento. }\end{array}$} & \begin{tabular}{|l} 
Equipo Auditor \\
Delegado del área de mantenimiento
\end{tabular} \\
\hline \multicolumn{3}{|c|}{ Revisado por: } & $\begin{array}{l}\text { Aprobado } \\
\text { por: }\end{array}$ \\
\hline
\end{tabular}


Fuente: (Chang, 2019)

De acuerdo con la figura $\mathrm{N}^{\circ} 4$, es posible conocer los criterios de planificación y programación de mantenimiento (PP), control de la gestión (CG) y recursos humanos de mantenimiento (RH); no cumplieron totalmente con los requisitos del cuestionario auditor. Respecto al criterio de organización de mantenimiento obtuvo la puntuación máxima de 20,86\%. Recursos humanos de mantenimiento alcanzó el 12,25\% siendo el valor máximo el 17\% debido a la presencia de una no conformidad en el subcriterio formación profesional.

El control de la gestión alcanzó el 17,87\% teniendo en cuenta que su nivel de referencia es del $20 \%$. El porcentaje que obtuvieron los criterios de planificación y programación del mantenimiento, mantenimiento correctivo, contratación externa de mantenimiento y manejo de inventario para bodega de mantenimiento fueron de $17.34 \%, 9.67 \%, 5.56 \%, 8.56 \%$ y los valores máximos a los que era posible llegar son de $18 \%, 10 \%, 6 \%$ y $8 \%$ respectivamente (Chang, 2019).

Figura $\mathbf{N}^{\circ}$ 4: Desempeño de los criterios de auditoría

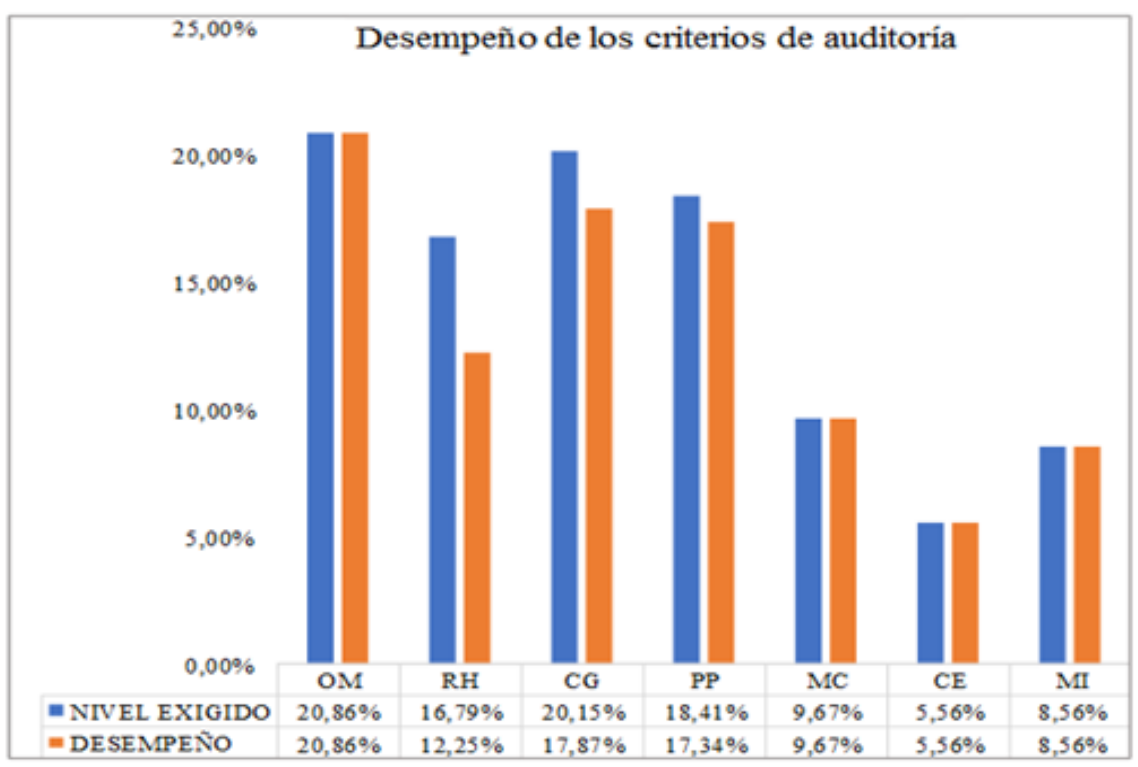

Fuente: Los autores

En la tabla $\mathrm{N}^{\circ} 7$, se indican los valores que obtuvo la empresa auditada en cada criterio; de forma global, cuantitativamente alcanzó el $92 \%$ del cumplimiento de requisitos para la gestión de mantenimiento, lo que equivale a un nivel cualitativo "cuasi satisfactorio" según esta metodología de auditoría. 
Tabla No 7: Valores por criterio de auditoría.

\begin{tabular}{|c|c|c|c|c|c|c|}
\hline REQUISITOS & $\begin{array}{l}\text { NIVEL EXI- } \\
\text { GIDO }\end{array}$ & $\begin{array}{l}\text { DEFI- } \\
\text { CIENTE }\end{array}$ & $\begin{array}{l}\text { POCO SATIS- } \\
\text { FACTORIO }\end{array}$ & $\begin{array}{l}\text { CUASI SATIS- } \\
\text { FACTORIO }\end{array}$ & $\begin{array}{l}\text { SATISFACTO- } \\
\text { RIO }\end{array}$ & $\begin{array}{l}\text { DESEM- } \\
\text { PEÑO }\end{array}$ \\
\hline $\begin{array}{l}\text { Organización de manteni- } \\
\text { miento }\end{array}$ & $20,86 \%$ & 0 & 7,3 & 14,6 & 20,86 & $20,86 \%$ \\
\hline $\begin{array}{l}\text { Recursos humanos de } \\
\text { mantenimiento }\end{array}$ & $16,79 \%$ & 0 & 5,88 & 11,75 & 7,49 & $12,25 \%$ \\
\hline $\begin{array}{l}\text { Control de la gestión de } \\
\text { mantenimiento }\end{array}$ & $20,15 \%$ & 0 & 7,05 & 14,1 & 15,48 & $17,87 \%$ \\
\hline $\begin{array}{l}\text { Planificación y programa- } \\
\text { ción de mantenimiento }\end{array}$ & $18,41 \%$ & 0 & 6,44 & 12,89 & 16,22 & $17,34 \%$ \\
\hline Mantenimiento correctivo & $9,67 \%$ & 0 & 3,38 & 6,77 & 9,67 & $9,67 \%$ \\
\hline $\begin{array}{l}\text { Contratación externa de } \\
\text { mantenimiento }\end{array}$ & $5,56 \%$ & 0 & 1,95 & 3,9 & 5,56 & $5,56 \%$ \\
\hline \multirow[t]{2}{*}{$\begin{array}{l}\text { Manejo de inventario para } \\
\text { bodega de mantenimiento }\end{array}$} & $8,56 \%$ & & 2,99 & 5,99 & 8,56 & $8,56 \%$ \\
\hline & TOTAL & & & & & $92 \%$ \\
\hline
\end{tabular}

Fuente: Elaboración propia

\section{Conclusiones.}

- Esta investigación ha determinado que los criterios más importantes que deben ser considerados en una auditoría de la gestión de mantenimiento de activos físicos son: organización $(21 \%)$, control de la gestión de mantenimiento $(20 \%)$, recursos humanos (17\%), planificación y programación (18\%), mantenimiento correctivo $(10 \%)$, manejo de inventario de bodega $(8 \%)$ y contratación externa de mantenimiento $(6 \%)$.

- La fase de validación de la metodología determinó un total de 65 hallazgos correspondientes a 62 conformidades y 3 no conformidades. El análisis de los resultados demostró que la gestión del mantenimiento alcanzó el 92\% de cumplimiento durante la auditoría que corresponde a un nivel cuasi satisfactorio.

\section{Referencias bibliográficas.}

Acosta, H., \& Troncoso, M. (2011). Auditoría integral de mantenimiento en instalaciones hospitalarias , un análisis objetivo. Ingeniería Mecánica, 14(2), 107-118

Aznar, J., \& Guijarro, F. (2012). Nuevos métodos de valoración: modelos multicriterio (2 Edición; Universitat Politècnica, Ed.). Valencia: Universitat Politècnica. 
Bana, C. A., Carmen, M., \& Duarte, M. (2012). A multi-criteria model for auditing a Predictive Maintenance Programme. European Journal of Operational Research, 217(2), 381-393. https://doi.org/10.1016/j.ejor.2011.09.019

Carnero, M. C., \& Gómez, A. (2016). A multicriteria decision making approach applied to improving maintenance policies in healthcare organizations. BMC Medical Informatics and Decision Making, 22. https://doi.org/10.1186/s12911-016-0282-7

CGE, (2003).Manual General de auditoría gubernamental, que regirá para las unidades de auditoría de la contraloría, de auditoría interna de las entidades y organismos del sector público que se encuentran bajo el ámbito de competen- cia del organismo técnico superior.Cholasuke,

Chang, M. (2019).Elaboración de un modelo de auditoría para evaluar la gestión de mantenimiento de activos físicos en base a normativa internacional aplicado al caso de estudio: Unión Cementera Nacional (UCEM) planta Chimborazo. Riobamba: Escuela Superior Politécnica de Chimborazo. http://dspace.espoch.edu.ec/handle/123456789/11472

C., Bhardwa, R., \& Antony, J. (2004). The status of maintenance management in UK manufacturing organisations: results from a pilot survey. 10(1). https://doi.org/10.1108/13552510410526820

COVENIN 2500. Manual para evaluar los sistemas de mantenimiento en la industria., (1993). Venezuela.

EN 13306:2018 Terminología de mantenimiento., (2018).España.

Espinosa, F. F., \& Salinas, G. E. (2010). Evaluación de la madurez de la función mantenimiento para implementar innovaciones en su gestión. Informacion Tecnologica, 21(3), 3-12. https://doi.org/10.1612/inf.tecnol.4313it.09

Galar, D., Kumar, U., Aditya, P., \& Berges, L. (2011). Auditorias de mantenimiento. (March).

Grencki, J., \& Legát, V. (2007). Maintenance audit and benchmarking - search for evaluation criteria on global scale. (3). ISO 19011. (2011). Directrices para la auditoría de Sistemas de Gestión (Vol. 2011). Vol. 2011.

Ginebra. Jiménez, S., \& Valencia, H. (2012). Sistema de gestión para mantenimiento de equipos eléctricos mediante indicadores de confiabilidad. Telecomunicaciones e Informática, 2(4), $11-23$.

ISO 19011 (2011). Directrices para la auditoría de Sistemas de Gestión. Suiza

Mora, L., \& Arango, M. (2010). Auditoría - Costos y nivel de mantenimiento en una organización. Eafit, 80. 
Mwanza, B., \& Mbohwa, C. (2015). An assessment of the effectiveness of equipment maintenance practices in public hospitals.

Procedia Manufacturing, 4(Iess), 307-314. https://doi.org/10.1016/j.promfg.2015.11.045 NB12017. Sistemas de gestión de mantenimiento - Requisitos. , (2013).

Orozco, W., Narváez, G., García, W., \& Quintero, A. (2017). Gestión de mantenimiento y producción más limpia en tres instituciones de salud de Medellín , Colombia. Revista Ingeniería Biomédica, 11(21), 21-25. https://doi.org/DOI: https://doi.org/10.24050/19099762.n21.2017.1168

Pecchia, L., \& Melillo, P. (2013). Multicriteria and Multiagent Decision Making with Applications to Economics and Social Sciences. Studies in Fuzziness and Soft Computing, Vol. 305, pp. 275-288. https://doi.org/10.1007/978-3-642-35635-3 Restrepo-Medina,

M. A. (2018). Calidad de los hallazgos de auditoría. Análisis de caso de las contralorías territoriales de Colombia. Innovar, 28(70), 115-128. https://doi.org/10.15446/innovar.v28n70.74452

Rodríguez Rieiro, C., Olmedo Lucerón, M. C., Flavia Rodríguez Gijón, L., Plá Mestre, R., Izquierdo Membrilla, I., Seriña Ramirez, C., \& Rodríguez Pérez, P. (2010). El programa de auditorías internas según norma UNE-EN ISO 9001 realizadas con personal propio: Herramienta para la gestión del conocimiento.

Revista de Calidad Asistencial, 25(3), 161-168. https://doi.org/10.1016/j.cali.2010.01.005

Sánchez, J., \& Calderón, C. (2013). Auditoría a la etapa de planificación y diseño del proceso de compensación. Estudios Gerenciales, 29, 139-150. https://doi.org/10.1016/j.estger.2013.05.002

Vaisnys, P., Contri, P., Rieg, C., \& Bieth, M. (2016). Monitoring the effectiveness of maintenance programs through the use of performance indicators Summary Report.

Viscaíno Cuzco, M. A., Villacrés Parra, S. R., Gallegos Londoño, C. M., \& Negrete Costales, J. H. (2019). Evaluación de la gestión del mantenimiento en hospitales del Instituto Ecuatoriano de Seguridad Social de la Zona 3 del Ecuador. Ingenius, (22), 59-71. https://doi.org/10.17163/ings.n22.2019.06

Viscaíno, M., Quesada, F., \& Villacrés, S. (2017). Priorización de criterios para la evaluación de la gestión del mantenimiento en edificios multifamiliares. Arquitectura y Urbanismo ISSN:, XXXVIII, 61-70. Retrieved from http://rau.cujae.edu.cu/public/Revistas Completas/2017/AU-3-2017.pdf Viveros,

P., Stegmaier, R., Kristjanpoller, F., Barbera, L., \& Crespo, A. (2013). Propuesta de un modelo de gestión de mantenimiento y sus principales herramientas de apoyo. Revista Chilena de Ingeniería, 21(1), 125-138. https://doi.org/10.4067/S0718-33052013000100011. 


\section{PARA CITAR EL ARTÍCULO INDEXADO.}

Chang Parrales, M. F., Villacrés Parra, S. R., Viscaíno Cuzco, M. A., \& Gallegos Londoño, C. M. (2020). Modelo de auditoría para evaluar la gestión de mantenimiento de activos físicos. ConcienciaDigital, 3(1.2), 104-122. https://doi.org/10.33262/concienciadigital.v3i1.2.1189

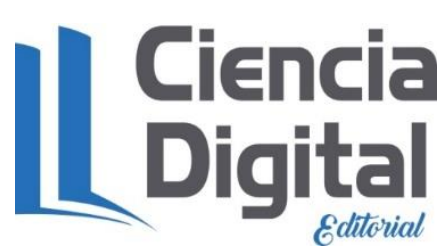

El artículo que se publica es de exclusiva responsabilidad de los autores y no necesariamente reflejan el pensamiento de la Revista Conciencia Digital.

El artículo queda en propiedad de la revista y, por tanto, su publicación parcial y/o total en otro medio tiene que ser autorizado por el director de la Revista Conciencia Digital.

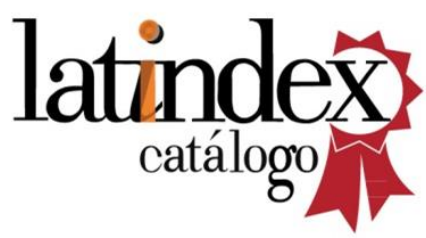

